\title{
Neighbouring Color Dependence Matrix for Image Analysis : Application to homogeneous and heterogeneous areas detection and characterization
}

\author{
B. Jacquin* and A. Smolarz* \\ * University of Technology of Troyes, ICD Laboratory FRE CNRS 2848, BP 2060 F10010, TROYES CEDEX, FRANCE \\ Received 12 may 2006; revised 4 september 2006; accepted 26 February 2008
}

\begin{abstract}
A new method for color texture characterization and color texture region detection is presented. This method, which we will name NCDM (Neighbouring Color Dependence Matrices), is the extension to color textures of the NGLDM (Neighbouring Gray Level Dependence Matrices) introduced by Sun et al. [1] and completed by Berry et al. [2]. This approach consists in estimating the dependences of colors between a pixel and its neighbours. We propose two steps: a color areas classification in two classes followed by the characterization of the detected areas. In the first step, we compute the NCDM with an isotropic neighbourhood. The structure of the isotropic NCD distribution allow us to separate the pixels of a color composite image into two classes, which correspond respectively to homogeneous and heterogeneous regions in the image. We then consider that the heterogeneous regions are potentially textured regions and in the second step we propose to compute the NCDM with anisotropic neighbourhoods corresponding to the eight principal directions. To seek the dominant directions in a color texture, a measure of spatial dependence between a pixel and its neighbours is computed by way of a chi-square test. This measure is based on the fit of the NGLD and NCD distribution with a binomial model under independence hypothesis. The variations of the colors are computed in uniform perceptual color spaces. We have chosen the color space "L1 norm" introduced by Angulo and Serra [3].
\end{abstract}

Key Words: Color space, Color Texture, Anisotropy, NGLDM, Color Image Segmentation, Chi-square Test.

\section{Introduction}

We propose a new method for the analysis of color images which is of important use in content-based image retrieval (CBIR) systems, composed of two principal steps. The first step consists in separating a color image into two classes. In order to do that, we classify all the pixels that satisfy a criterion of homogeneity (in terms of color) as belonging to a "homogeneous class". Let us specify that class relates to an area because our criterion of homogeneity is a spatial criterion with a local measure in the neighbourhood of a pixel. The non-labeled pixels do not satisfy the criterion of homogeneity that we propose, and these pixels can belong to textured areas, to noisy areas, or to the boundaries between the areas. The second step consists in extracting a set of features on these pixels in order to detect and to characterize the heterogeneous areas. The features are based on a measure

Correspondence to: <andre.smolarz@utt.fr>

Recommended for acceptance by Jean-Marc Ogier

ELCVIA ISSN:1577-5097

Published by Computer Vision Center / Universitat Autònoma de Barcelona, Barcelona, Spain 
of anisotropy in the eight principal directions. Our method is an extension of the Neighbouring Gray Level Dependence Matrices (NGLDM, Sun \& Wee in 1983 [1] and Berry \& Goutsias in 1991 [2]) to color images, which we will call : Neighbouring Color Dependence Matrices (NCDM). These matrices evaluate the degree and the nature of the local dependence in the neighbohood of a pixel. The definition of a NCDM matrix will be detailed in section 3. To evaluate the efficiency of the proposed method, we will use a publicly image database and we will give the number of correct classified pixels.

Initially, we will review the main works that deal with the definition of the color texture (section2). In section 3 , we recall the definition and the notations used with NGLD matrices. We also present the measures of homogeneity and heterogeneity that we propose for the first step of classification. Then we define the measure of anisotropy and the following set of features used for the second step of detection and characterization of texture. Finally, this section is completed with the extension of the definition of NGLD matrices to NCD matrices and we discuss the consequences of applying the methodology to color images.

In section 4, we present the first step of separation of the pixels in two classes corresponding to the homogeneous and heterogeneous areas. Some examples will illustrate this first step which is computed in RGB color space.

The second aspect of our paper is presented in section 5. By considering that the heterogeneous areas are potentially textured areas, we calculate the NCD matrices associated with anisotropic neighbourhoods corresponding to the eight principal directions. Some examples will illustrate this second step which is computed in "L1 norm" color space ([3] and [7]). Finally, we will present our conclusion and the prospects for future work.

\section{Previous works}

We describe here works on color textures. Firstly, we will discuss the perceptual properties that can be observed when a texture is examined.

Rao and Lohse [9] reported the results of an experiment in which the aim was to find the high level characteristics of the texture for human vision. Twenty people were asked to independently classify 30 textures of the Brodatz album [12]. The authors identified the most significant characteristics as the regularity (a pattern which is repeated), followed by the orientation and the complexity.

Mojsilovic et al. [13] presented the results of a similar experiment with color textures. They asked 28 subjects to specify numerically, on a scale from 0 to 100 , the similarities between each possible combination of 20 color textures extracted from a set of fabrics. After a statistical analysis, they identified five significant perceptual characteristics. Two characteristics were specific to the color: the dominant color and the purity of the color. The three other characteristics corresponded to the characteristics found by Rao and Lohse: directionality and orientation, regularity, complexity and density. The NCD matrices, which are presented in the next section, implicitly provide these features : the dominant color, the orientation and the structure of the textures.

Angulo, Hanbury and Serra in [5], [4] and [3] have worked with oriented textures for studies on the automatic classification of wood according to two criteria: the color and the texture. They sought the dominant directions in the neighbours of each point of the image. Serra and Hanbury used the algorithm proposed by Rao ([10] and [11] ) to find these directions. The first step of Rao's algorithm ( [10] and [11] ) applies a Gaussian filter to the texture. It is necessary to calculate an angle for each pixel with the images for horizontal and vertical gradient. The dominant angle is calculated in the neighbourhoods in order to produce an angle image. Each pixel in the angle image corresponds to the dominant direction in a neighbourhood.

Germain et al. [14] worked on the characterization of the anisotropy of the textured images. In their works, they reported that the dominant direction or the anisotropy in the textures strongly depends on the observation scale. They introduced a new operator for the estimation of the dominant direction, the Directional Mean Vector $(D M V)$, which works only on gray level textures. With the NCD matrices, different sizes of the neighbourhood enable a multi-scale analysis to be used in order to characterize the anisotropy in the color textures.

Shu and Jain [15] presented a method based on the properties of vector fields for the estimation of a set of symbolic descriptors from linear orientation fields. The authors presented results of experiments on real oriented 
textures.

Almeida [16] presented a Markov random field (MRF) model for digital images that were able to represent anisotropic textures with arbitrary orientations.

Works relating to the characterization of textures in gray levels with NGLD matrices were proposed by Smolarz in 2003 in [6]. An extension to color textures of these works is presented in section 5 of the current work.

Fontaine et al. [17] presented tools for spatial and colorimetric analysis that take into account the global and local colorimetric information of an image, such as the color co-occurrence matrices and the color correlograms initially introduced by Huang et al. [18].

\section{Presentation of the NGLDM and NCDM}

In order to clearly present the criteria that we have defined on the NCDM, we will recall some notations and definitions related to the NGLDM.

\subsection{Notations used for the NGLDM}

$N_{G} \quad$ Maximum number of gray levels.

$G(\ell, c) \quad$ Random variable that represent the gray level of the pixel $(\ell, c) . G \in\left\{0,1, \ldots N_{G}-1\right\}$

$d \quad$ Parameter equal to the maximum distance (in pixels) between the central pixel and its neighbours.

$V_{d} \quad$ The set of neighbours, called the neighbourhood.

$N_{d} \quad$ The number of neighbours. $N_{d}=\#\left\{V_{d}\right\}$

$a \quad$ The threshold of the difference between the gray levels of the central pixel and one of its neighbours.

$S \quad$ Random variable that represents the number of neighbours which have a gray level "close to" (as defined by parameter $a$ ) that of the central pixel. $S \in\left\{0,1, \ldots N_{d}\right\}$

In the original version of Sun et al. [1], the parameters $d$ and $a$ of the NGLDM are fixed by the user. In the current application, the parameter $a$ will be fixed automatically for the NCD matrices (see section 4), which reduces the need for user input, as well as providing greater objectivity.

\subsection{Definition of the NGLDM}

In order to simplify the notations, the NGLDM will be noted as $Q$. The general term of $Q$ is given by the expression:

$$
Q_{d, a}(g, s)=\#\left\{(\ell, c) \mid G(\ell, c)=g \text { and } \#\left[(i, j) \in V_{d}|| G(\ell, c)-G(i, j) \mid \leq a\right]=s\right\}
$$

Hence the NGLDM is a matrix of size $N_{G} \times\left(N_{d}+1\right)$ and $Q_{d, a}(g, s)$ is the number of pixels in an image with gray level $g$ that have exactly $s$ neighbours with gray level in the interval $[g-a, g+a]$.

The matrix $Q$ allows us to calculate the estimated joint distribution of the random variables $G$ and $S$, as shown below:

$$
\hat{P}_{d, a}[G=g, S=s]=\frac{Q_{d, a}(g, s)}{\sum_{u=0}^{N_{G}-1} \sum_{v=0}^{N_{d}} Q_{d, a}(u, v)}
$$

\subsection{Homogeneity, heterogeneity and NGLDM}

Firstly, in order to identify the homogeneity, we make the same assumptions as Deng et al. [19]: each image contains a set of approximately homogeneous color-texture regions. The color information in each image region can be represented by a set of a few quantized colors. The colors between two neighbouring regions are distinguishable. A region is called heterogeneous region if each pixel and its surrounding neighbours have 
different colors.

If we consider only the first columns of $Q$, it is possible to find the gray levels that best characterize the heterogeneous regions. Conversely, if we consider the last columns of $Q$, it is possible to find the gray levels that best characterize the homogeneous regions.

For example, consider the gray level $g$ : if for $n_{1} \ll N_{d}$ we have a high value of $\sum_{v=0}^{n_{1}} \hat{P}_{d, a}[G=g, S=v]$ we can conclude that the gray level $g$ lies in a heterogeneous region. If for $0 \ll n_{2}<N_{d}$ we have a high value of $\sum_{v=n_{2}}^{N_{d}} \hat{P}_{d, a}[G=g, S=v]$ we can conclude that the gray level $g$ lies in a homogeneous region.

Our approach to distinguish between homogeneous and textured regions is based on these properties of the NGLDM. The method will be detailed and illustrated in section 4.

\subsection{The binomial model and the anisotropy measure}

According to the previous formula (2), we can define the following conditional probability estimation:

$$
\hat{P}_{d, a}[S=s \mid G=g]=\frac{Q_{d, a}(g, s)}{\sum_{v=0}^{N_{d}} Q_{d, a}(g, v)}
$$

Under the non realistic (especially in the case of texture modeling) hypothesis that the random variables $\left\{G(\ell, c) \mid(\ell, c) \in V_{d}\right\}$ are independant, the distribution $\hat{P}_{d, a}[S=s \mid G=g]$ estimated by (3) is a binomial distribution with parameters $N_{d}$ and $p=P[g-a \leq G \leq g+a]$. We have choosen to use the well known Chi-Square distance to test the independence hypothesis. The resulting criterion is computed as follows:

1. For each gray level $g$, compute the Chi-Square distance :

$$
E(g)=\sum_{s=0}^{N_{d}} \frac{\left(Q_{d, a}(g, s)-Q_{d, a}(g, \cdot) \hat{P}_{d, a}[s \mid g]\right)^{2}}{Q_{d, a}(g, \cdot) \hat{P}_{d, a}[s \mid g]} \quad \text { where } \quad Q_{d, a}(g, \cdot)=\sum_{s=0}^{N_{d}} Q_{d, a}(g, s)
$$

2. For a fixed type one error $\alpha$ (typically $5 \%$ ), calculate the difference $D(g)=E(g)-\chi_{\alpha}^{2}$ between the ChiSquare distance and the test threshold at level $\alpha$.

3. Finally compute the mean value

$$
D=\frac{1}{N_{G}} \sum_{g=0}^{N_{G}-1} D(g)
$$

If $D$ has a negative value, we can accept the independence hypothesis. In the case of a positive value for $D$, the greater the value of $D$, the stronger the dependence hypothesis.

To take the anisotropy of textures into account, we can compute many NGLDM defined on anisotropic neighbourhoods, which are simply lines. Consider, for example, the four principal directions $0^{\circ}, 45^{\circ}, 90^{\circ}$ and $135^{\circ}$. We obtain four values $D_{0}, D_{45}, D_{90}$ and $D_{135}$ of the criterion defined by (4). $\left\{D_{0}, D_{45}, D_{90}, D_{135}\right\}$ constitutes a set of features that can be used to measure the anisotropy of a texture. In order to have normalized features, we propose to use the set $\left\{f_{0}, f_{45}, f_{90}, f_{135}\right\}$ defined by:

$$
f_{\theta_{i}}=\frac{D_{\theta_{i}}}{\sum_{j}\left|D_{\theta_{j}}\right|} \quad \text { and so } \quad f_{\theta_{i}} \in[-1,1] \quad \forall \theta_{i}=0^{\circ}, 45^{\circ}, 90^{\circ}, 135^{\circ}
$$

In the case of independence (for example a uniform or gaussian noise), all the features $f_{\theta_{i}}$ will have the theoretical value -0.25 , whereas in the case of an isotropic texture, all the features will have values close to 0.25 . These properties have been tested, with full details available in [6] 


\subsection{Definition of the NCDM}

We will now investigate how to define a Neighbouring Dependence Matrix based on color images. If we refer to formula (1), the main problem comes from the expression $|G(\ell, c)-G(i, j)| \leq a$. The question is how can the difference between two colors be calculated?

The answer that we propose is, according to a pre-defined color space, to use a distance between two colors that is called Perceptual Difference PD (see [4]). The Perceptual Difference can be defined as follows. We consider two color spaces, the cartesian space $R G B$ and the cylindrical coordinate space $H B S$. The gray level random variable $G(\ell, c)$ in formula (1) will be substituted by a random color variable $C(\ell, c)=$ $[R(\ell, c), G(\ell, c), B(\ell, c)]$ in the cartesian color space and $C(\ell, c)=[H(\ell, c), B(\ell, c), S(\ell, c)]$ in the cylindrical coordinate space. First of all, let us introduce some simple notations that will be used afterwards. Let $C_{1}=C\left(\ell_{1}, c_{1}\right)$ and $C_{2}=C\left(\ell_{2}, c_{2}\right)$ be the colors of two pixels $\left(\ell_{1}, c_{1}\right)$ and $\left(\ell_{2}, c_{2}\right)$ then, $\operatorname{PD}\left(C_{1}, C_{2}\right)$ is defined by:

$$
\begin{aligned}
& P D\left(C_{1}, C_{2}\right)=\sqrt{\left(R_{1}-R_{2}\right)^{2}+\left(G_{1}-G_{2}\right)^{2}+\left(B_{1}-B_{2}\right)^{2}} \quad(R G B \text { space }) \\
& P D\left(C_{1}, C_{2}\right)=\sqrt{\left(B_{1}-B_{2}\right)^{2}+S_{1}^{2}+S_{2}^{2}-2 S_{1} S_{2} \cos \left(H_{1}-H_{2}\right)} \quad(H B S \text { space })
\end{aligned}
$$

According to the definitions given by the formulas (6) and (7), we can now define a new matrix $Q$ which we call NCDM with the general term:

$$
Q_{d, a}(h, s)=\#\left\{(\ell, c) \mid C(\ell, c)=h \text { and } \#\left[(i, j) \in V_{d} \mid P D(C(\ell, c), C(i, j)) \leq a\right]=s\right\}
$$

It should be noted that $h$ in formula (8), represents a color triplet rather than a scalar as is the case for $g$ in formula (1).

Let us clarify our model for the measure of the anisotropy in a color image: $\hat{P}_{d, a}[S=s \mid C=h]$ is the estimated distribution, conditionally with the color of a pixel, of the number $s$ of its neighbours having a close color (the perceptual difference $P D$ is lower than $a$ ). Always under the assumption that the pixels belonging in the neighbourhood are independent, the probability $\hat{P}_{d, a}[S=s \mid C=h]$ can be modeled by the binomial distribution with parameters $N_{d}$ and $p=P[D P \leq a]$.

The test is carried out by computing the Chi- 2 distance for each color $h$ with $p$ dependent on $h$ [7]. Then, we compute the difference $D(h)$ between the Chi-2 distance and the decision threshold $\chi_{\alpha}^{2}$, obtained for a fixed type one error, in this case $\alpha=5 \%$.

In order to take the color distribution into account, we calculate the mean of the differences $D(h)$ balanced by the frequency (see formula (9)) instead of the arithmetic mean suggested with the formula (5). We compute this mean for each direction $\theta_{i}$.

$$
D_{\theta_{i}}=\sum_{h=0}^{N_{h}-1} D(h) f(h) \quad \text { where } \quad f(h)=\sum_{v=0}^{N_{d}} \hat{P}_{d, a}[C=h, S=v]
$$

where $f(h)$ represents the frequency of the color $h$.

Finally, as for gray level images, we compute the standardized features according to formula (5). Negative values of $f_{\theta_{i}}$ indicate that there is no dependence between the pixels in the selected direction. The larger the positive coefficients $f_{\theta_{i}}$, the greater the dependence between the pixels.

\subsection{Color reduction and consequences on NCD matrices}

The size of a NCD matrix is $N_{h} \times\left(N_{d}+1\right)$ where $N_{h}$ is the number of colors in the image. A composite image can have several thousands of colors, and as for the co-occurrence matrices, we need to reduce the number 
of colors in order to avoid the estimation problems of NCD distribution. This quantification step of colors is critical for the separation step of the pixels into two classes corresponding to the homogeneous and the heterogeneous areas. Indeed, the variations of contrast and hue must not be amplified during this phase, particulary for homogeneous areas and spectrally homogeneous regions (reflections, shadows).

For optimal results, the quantification step proceeds after having changed color space in order to exploit the colorimetric properties of the new color space. After several tests of color reduction methods [20], we have retained the minimum variance quantization algorithm, which creates a new pallete of colors. After this algorithm, there is a spatial quantification step to smooth the contrast areas. With this algorithm, we can choose the number of colors in the new palette.

In the separation step of the pixels into homogeneous areas and heterogeneous areas, we reduce the composite image to 16 colors in order to have a good compromise between the estimation constraints of NCDM and the degradation of the homogeneous areas. This choice implies the assumption that there is, at most, 16 different homogeneous areas in a composite image, which seems to be a reasonable assumption. Let us specify that the number of colors is a parameter. We also tested the two steps by reducing the images to 32 colors. The results are similar and are presented on figure 3 .

For the next step of anisotropy characterization in the potentially textured areas, we reduce the remaining areas to 64 colors, because the texture characterization requires a higher level of details in the colors.

In order to work on estimated probabilities, NCD matrices are normalized according to formula (2) and with the notations of the formula (8). The construction of an NCD matrix depends on two parameters (see section 3 ): the parameter $d$, which gives the size of the neighbourhood and the parameter $a$, which is the threshold of the difference in color between two neighbouring pixels. The $a$ value changes according to the analyzed image and thus, we propose to determine it automatically for each image. After color reduction of an image, we calculate the histogram and then, the $a$ value is fixed automatically by seeking the mean of the perceptual distances $(P D)$ for all the color couples. This mean is computed as follows.

$$
a=\frac{\sum_{u=0}^{N_{h}-1} \sum_{v=0, v \neq u}^{N_{h}-1} P D(u, v) f(u)}{N_{h}\left(N_{h}-1\right)}
$$

In the two steps of this paper, we use a very interesting property of NCD matrices : computing a NCD matrix on an image which contains thousands of colors with an adequate value for $a$ is equivalent to computing a NCD matrix with a reduced color number and a larger value for $a$. Indeed, increasing the $a$ value is equivalent to summing the information of several lines of NCD matrix, and therefore, merging several colors. Thus, NCD matrices are compatible with a color reduction step.

\section{Separation step between homogeneous and heterogeneous regions}

As indicated previously, for this step, we reduce the analyzed image to 16 colors. We choose an isotropic neighbourhood with a size $d=2\left(N_{d}=24\right.$ neighbours). Thus, the NCD matrix has a size of $16 \times 25$.

Let us take for example the "Mandrill" image (presented on figure 2.a) reduced to 16 colors. This image can be seen as the fusion, or in other words the union, of 16 color labeled layers $I(h)$. Each color labeled layer $I(h)$ corresponds to 1 color among the 16 colors (see figure 1). These color labeled layers $I(h)$ are complementary, and form a partition on the set of the pixels of the initial image. Our goal is to sort these color labeled layers $I(h)$ from the most homogeneous to the most heterogeneous.

In section 3.3, we introduced two thresholds $n_{1}$ and $n_{2}$. We chose here $n_{1}=4$ and $n_{2}=20$, which means that the areas where a pixel has at most four neighbours of close color $\left(\approx 20 \%\right.$ of $\left.N_{d}\right)$ are labeled as heterogeneous and the areas where a pixel has at least twenty neighbours of close color $\left(\approx 80 \%\right.$ of $\left.N_{d}\right)$ are labeled homogeneous. Thus, for a given color $h$, if $\hat{P}_{d, a}[C=h, S=s]$ has a maximum in the left hand part of the matrix $\left(s \leq n_{1}\right)$, then $h$ is mainly present in the heterogeneous areas of the image. Conversely, if 
$\hat{P}_{d, a}[C=h, S=s]$ has a maximum in the right hand part of the matrix $\left(s \geq n_{2}\right)$, then $h$ is mainly present in the homogeneous areas of the image.

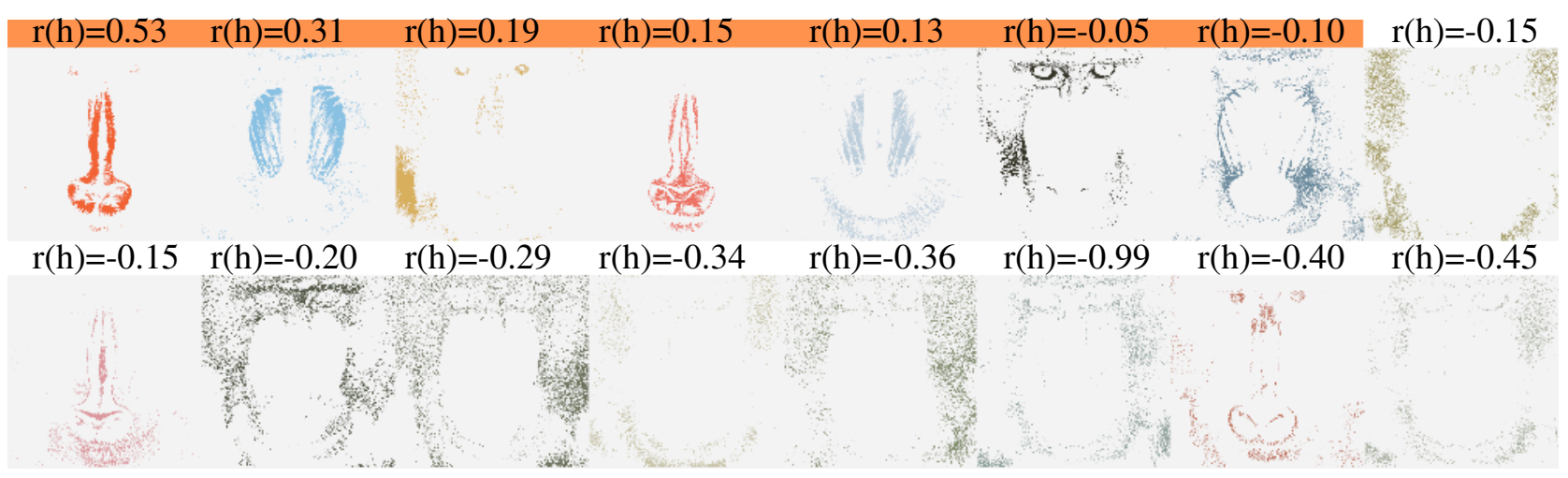

Figure 1: Layers of a "Mandrill" sorted from the most homogeneous one to the most heterogeneous one. The $r(h)$ values for each color labeled layer is displayed above it

Then, for each color $h$, that is to say for each color labeled layer $I(h)$, we compute the estimated probability $h e t(h)$ that a color belongs to a heterogeneous area and the estimated probability hom $(h)$ that a color belongs to a homogeneous area according to the formulas (11) and (12).

$$
\begin{gathered}
\operatorname{het}(h)=\hat{P}_{d, a}\left[C=h, S \leq n_{1}\right]=\sum_{v=0}^{n_{1}} \hat{P}_{d, a}[C=h, S=v] \\
\operatorname{hom}(h)=\hat{P}_{d, a}\left[C=h, S \geq n_{2}\right]=\sum_{v=n_{2}}^{N_{d}} \hat{P}_{d, a}[C=h, S=v]
\end{gathered}
$$

In order to work with a relative and standard measure, we compute a ratio $r(h)$ as follows.

$$
-1 \leq r(h)=\frac{\operatorname{hom}(h)-\operatorname{het}(h)}{f(h)} \leq+1 \quad \text { where } f(h) \text { represents the frequency of the color } h
$$

The color labeled layers are then sorted according to the values $r(h)$ with decreasing homogeneity (figure 1). It should be noted that the size of the homogeneous or heterogeneous areas (that is to say the number of pixels for each color labeled layer) does not influence this classification because the terms in the NCD matrix are normalized (see formula (2)).

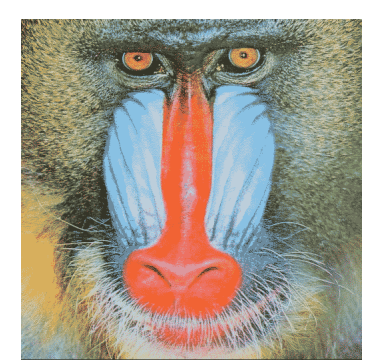

(a) original image

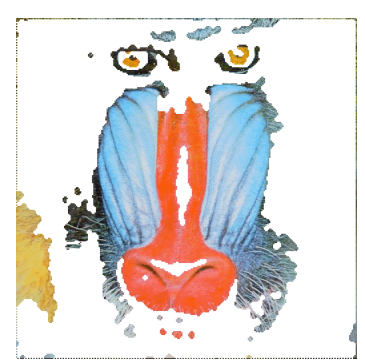

(b) homogeneous areas

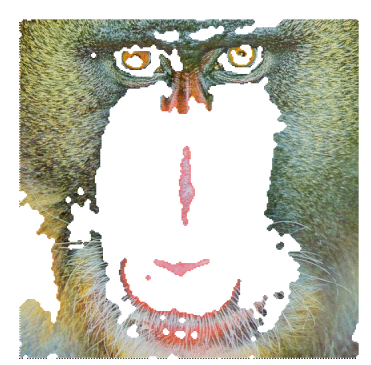

(c) heterogeneous areas

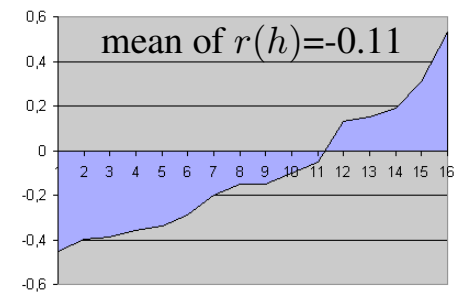

(d) profile of $r(h)$

Figure 2: An image of a "Mandrill" with 16 colors. The homogeneous and heterogeneous areas in (b) and (c) result from the threshold $a$ obtained from figure 1 


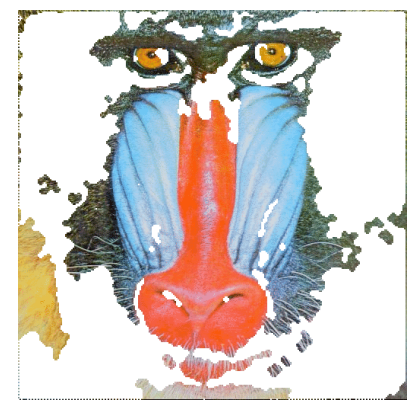

(a) homogeneous areas

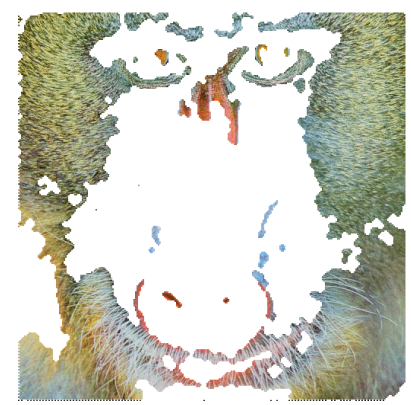

(b) heterogeneous areas

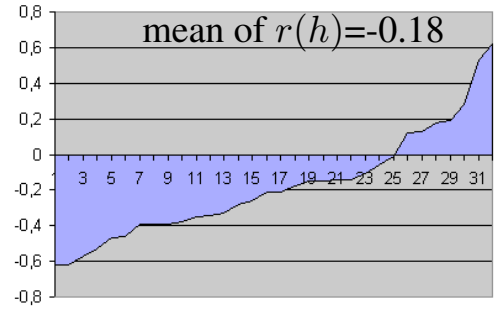

(c) profile of $r(h)$

Figure 3: An image of a "Mandrill" with 32 colors. The homogeneous and heterogeneous areas in (a) and (b) were obtained with a threshold $a$ equal to -0.18

From now on, we have to find a threshold $T$ for $r(h)$ such that the fusion of the color labeled layers $I(h)$ with $r(h) \leq T$ forms the set of the potentially textured areas and the fusion of the color labeled layers $I(h)$ with $r(h)>T$ forms the set of the homogeneous areas of the image. Experimentally, we have noticed that the arithmetic mean value of $r(h)$ seams to be a good threshold to separate between homogeneous and heterogeneous areas.

A profile of $r(h)$ appears on figure 2.d ( $h$ is on the X-axis and $r(h)$ on the Y-axis). We can see that the mean value is located towards the zero-crossing of this curve. So an other possible choice of the threshold $T$ can be the value $h$ of zero-crossing. The results of our experiments indicated that the mean value of $r(h)$ gives better classification results between hom $(h)$ and het $(h)$ on the test set.

Remembering that the color labeled layers have been sorted in decreasing order (according to $r(h)$ ), the threshold $T$ corresponds to the color $h$ of the less homogeneous layer $I(h)$, i.e. the layer with the value of $r(h)$ closest to $T$.

For the example of figure 1, the arithmetic mean value of $r(h)$ is -0.11 . Therefore, the color labeled layer corresponding to the threshold will be the one with $r(h)=-0.10$. This selected layer will be merged with all the color labeled layers more homogeneous than it (indicated by an orange stripe above them in figure 1), to form the set of homogeneous areas of the image "Mandrill" (figure 2.b). The other color labeled layers are merged, in order to form the set of heterogeneous (potentially textured) areas, which correspond to the fur of the Mandrill (figure 2.c). The final result is a partition of the original image into two classes : the homogeneous and the heterogeneous areas.

To illustrate the separation step of the pixels into two classes, we present, in figure 4, the results obtained on an image of a rabbit (the fur is well isolated), a carpark (the trees are well isolated), a golf course (the vegetation and its reflection in the water are identified as heterogeneous), some flowers (the flowers are well isolated) and a human face (the skin is well isolated). We can see that an heterogeneous area can be composed of a frontier between two homogeneous areas or a degraded gray-level. Let us note that this step of separation is very precise since the computation is made at a pixel level, thus enabling very fine edges to be obtained (see, for example, the tomato in the image of the rabbit).

This first step, from the loading of the image, thought the analysis until the creation of the two classes, requires less than one second of computing time for images of $512 \times 512$ pixels.

In order to evaluate in a quantitative way the efficiency of our approach, we have generated four synthetic images with known homogeneous and heterogeneous regions (see figure 5). The heterogeneous areas are composed of textures downloaded from the image database available at http://textures. forrest.cz/. In the four examples the background forms the homogeneous areas. The performance criterion is the rate of correct classified pixels calculated after the detection step of the two classes. For the first three images (figures 5.a, 5.d and 5.g), the percentages of correct classified pixels are respectively $94.4 \%, 99.7 \%$ and $97.7 \%$. These values, 


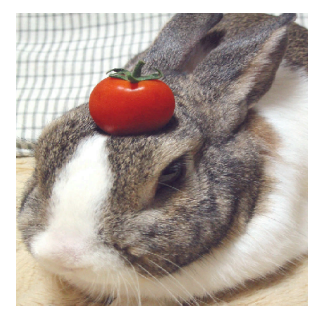

original image

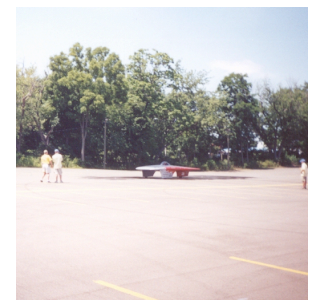

original image

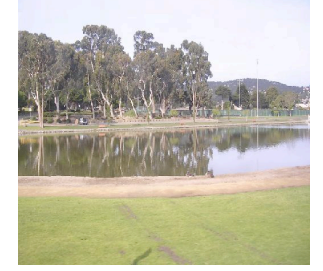

original image
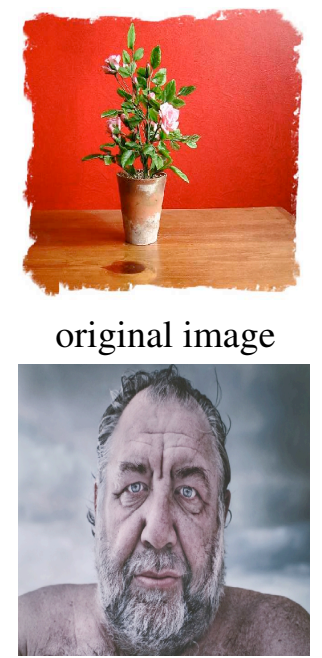

original image

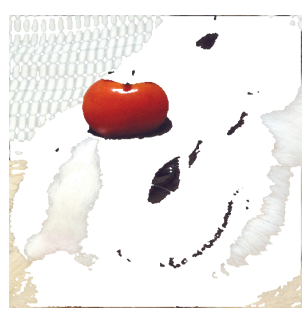

homogeneous areas

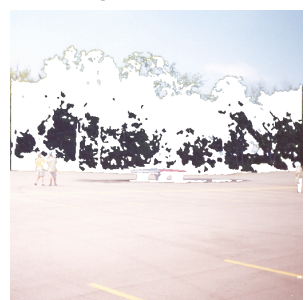

homogeneous areas

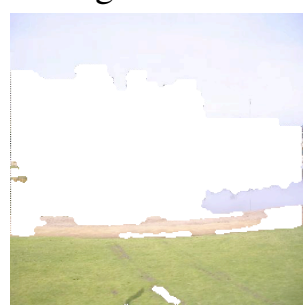

homogeneous areas

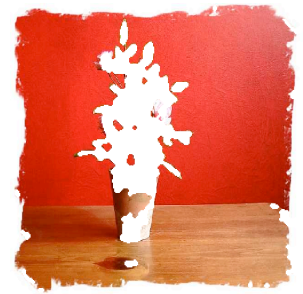

homogeneous areas

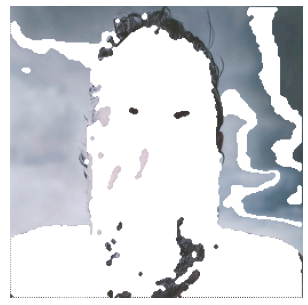

homogeneous areas

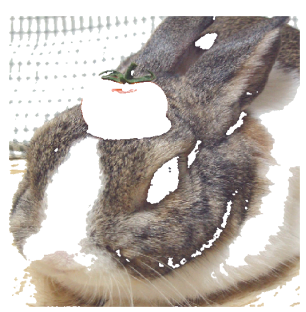

heterogeneous areas

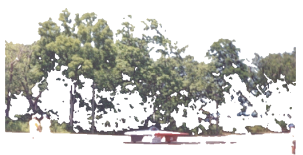

heterogeneous areas

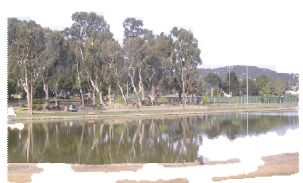

$\checkmark$

heterogeneous areas

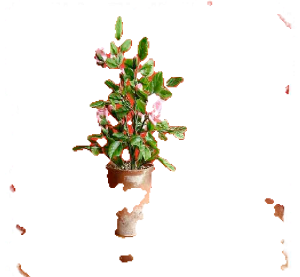

heterogeneous areas

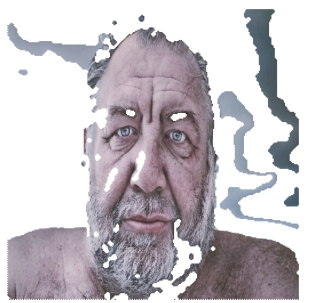

heterogeneous areas

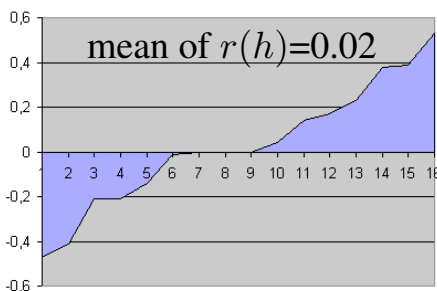

profile of $r(h)$

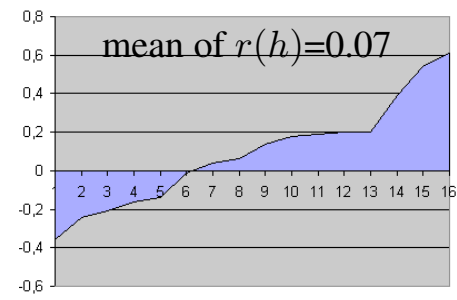

profile of $r(h)$

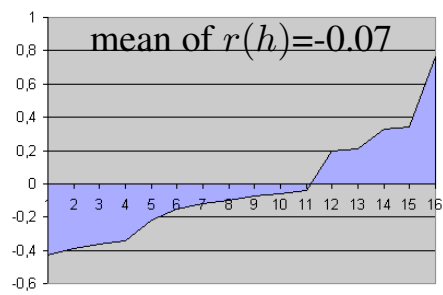

profile of $r(h)$

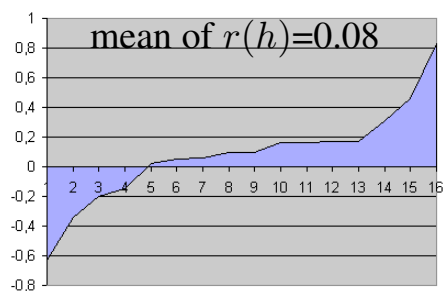

profile of $r(h)$

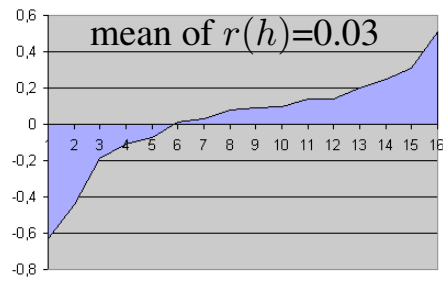

profile of $r(h)$

Figure 4: Separation of the pixels into two classes : homogeneous areas and heterogeneous areas, and the profiles of $r(h)$ 


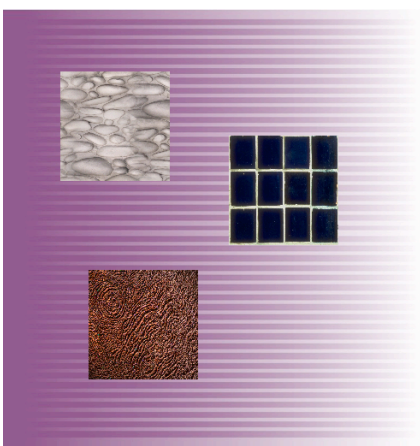

(a) test image 1

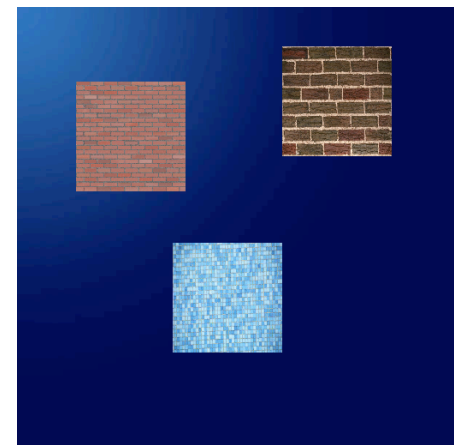

(d) test image 2

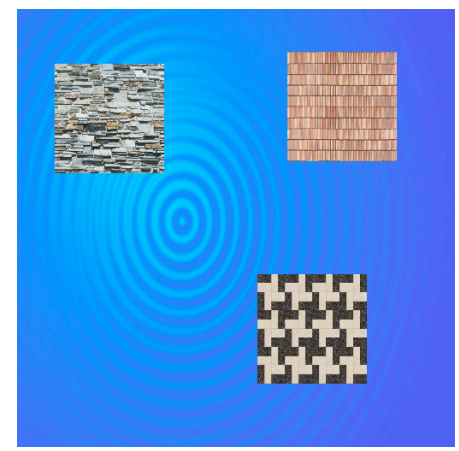

(g) test image 3

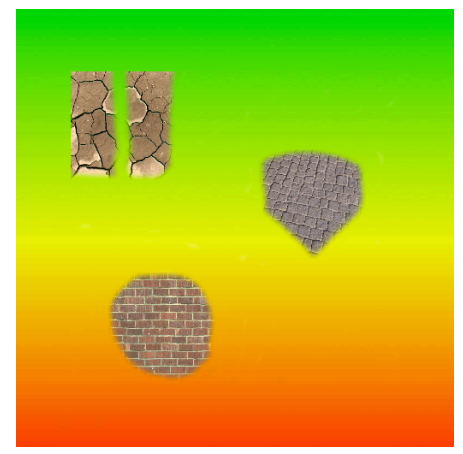

(j) test image 4

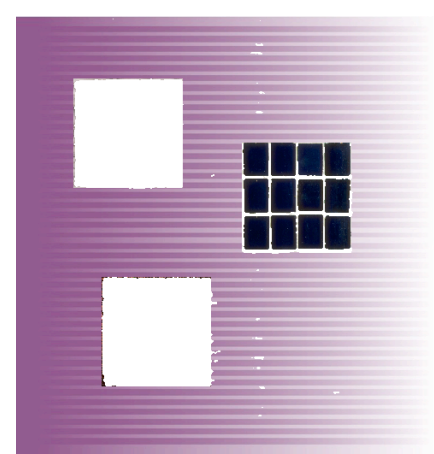

(b) homogeneous areas

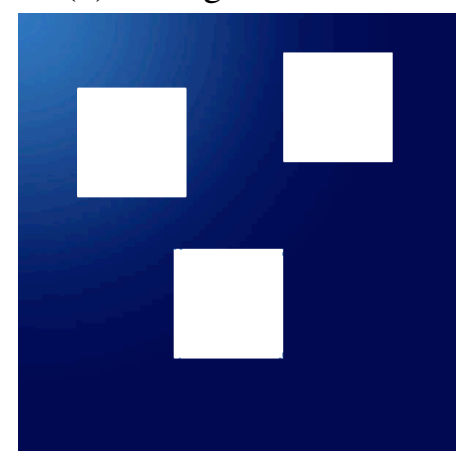

(e) homogeneous areas

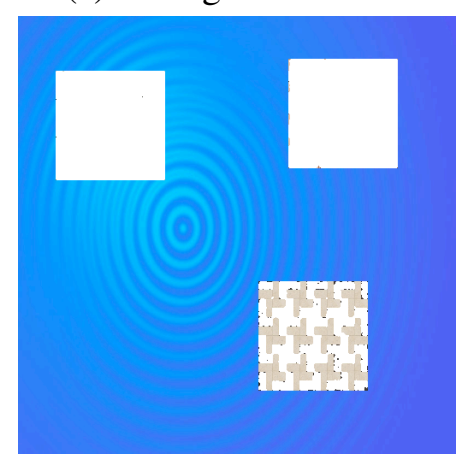

(h) homogeneous areas

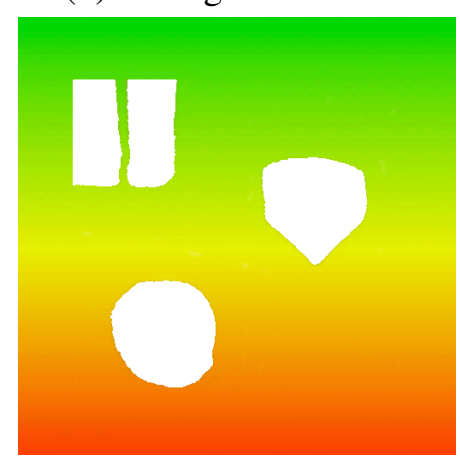

(k) homogeneous areas

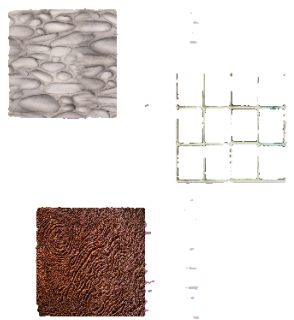

(c) heterogeneous areas

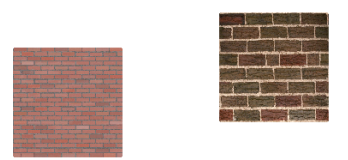

(f) heterogeneous areas
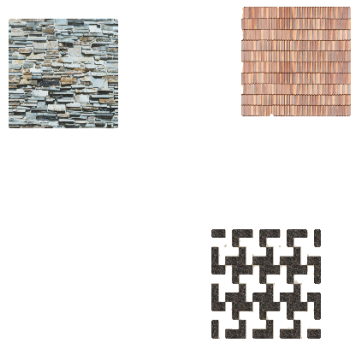

(i) heterogeneous areas
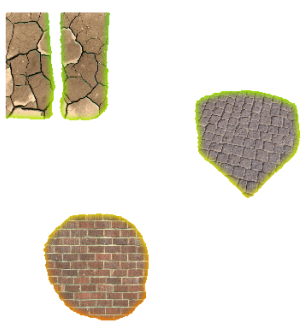

(1) heterogeneous areas

Figure 5: Detection of homogeneous and heterogeneous areas on synthetic images 
associated to the good visual results of the classification, show the efficiency of our method.

The fourth example (figure 5.j) is quite different because of the complexity of the boundaries between the heterogeneous and homogeneous areas. The percentage of correct classified pixels is $99.4 \%$. We can observe that the result is in this case still very good. This result is very interesting because on the figure 5.1, we can see that the detected heterogeneous areas are composed of the textures and also of the frontiers between the homogeneous and the heterogeneous areas.

So, we can conclude that our classification step provides very good results in terms of detection and localization of homogeneous and heterogeneous areas in color images. Unfortunately we cannot compare our method with other works because we have not found any paper related to this approach in the field of color image analysis.

\section{Characterization step of color texture by a measure of anisotropy}

After the classification step of the pixels, which enabled initial image to be partitioned, we keep the coordinates of the pixels of the potentially textured areas. The colors of the pixels of the original image are converted in the "L1 norm" space (although, it is possible to use another color space), which is a uniform color space introduced by Serra [3]. Our previous works [8] provided the evidence of effectiveness of the "L1 norm" for a second order method of color texture characterization. "L1 norm" space is a representation of color space RGB with polar coordinates. It is a space of the type : (brightness, hue, saturation). The parameters of intensity (brightness and saturation) are norms and are independent. "L1 norm" space is adapted for quantitative applications and thus, it seems well suited to the NCD matrices. For more information, the reader can refer to [3] [4] [5] [7].

Afterwards, the color image is reduced to 64 colors. This number of colors seems sufficient, according to our experiments, to characterize color textures. We compute the value of the parameter $a$ which was modified following the change of color space and the increase of the color number.

From now on, we use an anisotropic neighbourhood with a distance $d=4$ (i.e. four pixels around the central pixel in the selected direction) according to the eight principal directions : $0^{\circ}, 22.5^{\circ}, 45^{\circ}, 67.5^{\circ}, 90^{\circ}, 112.5^{\circ}$, $135^{\circ}$ and $157.5^{\circ}$. Therefore, we compute eight NCD matrices with a size $64 \times 9$.

The measure of independence between the pixels and the characterization of the anisotropy are based on a Chi-2 test (presented in sections 3.4 and 3.5). This method had initially been proposed by Smolarz in [6] for gray level textures, and subsequently extended to color textures [7]. This model enables independence to be evaluated by the features $f_{\theta_{i}}$ defined with the formulas (5) and (9).

On a texture of $128 \times 128$ pixels, the computing time to calculate eight NCD matrices and the Chi- 2 tests is around one second.

In section 3.4, we said that, in the case of independence, the features $f_{\theta_{i}}$ must be negative. This can be checked with a color texture composed of only independent gaussian noise (image $T_{1}$ on figure 6.a). Indeed, in table 1, we can see that, for the eight directions, the results are negative. They are almost identical and all the values $f_{\theta_{i}}, i=1,8$ are close to $-1 / 8$.

Table 1 gives the values of $f_{\theta_{i}}, i=1,8$ for the four textures of figure 6. $T_{2}$ is an isotropic texture, $T_{3}$ has a vertically dominant direction and $T_{4}$ has a horizontally dominant direction.

We note that, for texture $T_{2}$, we have approximately equal values for all the directions, only the directions $45^{\circ}$ and $135^{\circ}$ are slightly different. For texture $T_{3}$, we observe very large values for the direction $90^{\circ}$ and the close directions $67.5^{\circ}$ and $112.5^{\circ}$. For the other directions, the values are almost zero. The results for texture $T_{4}$ are similar with only $0^{\circ}, 22.5^{\circ}$ and $157.5^{\circ}$ having large coefficients.

These results show that the use of NCDM based on anisotropic neighbourhoods, associated with a statistical measure of independence, provides an accurate set of color texture features.

In order to confirm the efficiency of the method we present in table 3 the results of anisotropy measures obtained on a set of 21 images (image database http://textures. forrest.cz/) presented on figure 8.

From now on, we will apply this method on the areas of the Mandrill obtained after the classification step of the pixels (figure 2.b and 2.c). 


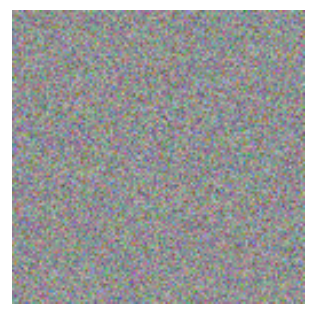

(a) $T_{1}$ : gaussian noise

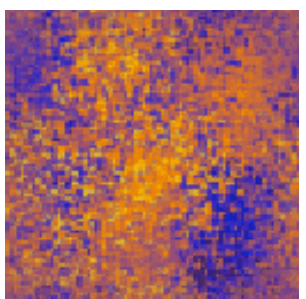

(b) $T_{2}$ : bright paper

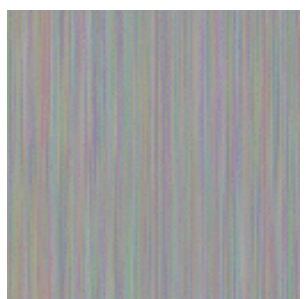

(c) $T_{3}$ : brushed aluminium

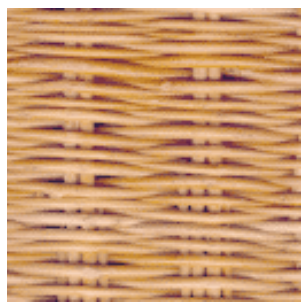

(d) $T_{4}$ : basket

Figure 6: Measure of independence between the pixels

\begin{tabular}{|l|c|l|c|l|c|l|l|l|}
\hline$d=4$ & $0^{\circ}$ & $22.5^{\circ}$ & $45^{\circ}$ & $67.5^{\circ}$ & $90^{\circ}$ & $112.5^{\circ}$ & $135^{\circ}$ & $157.5^{\circ}$ \\
\hline$T_{1}$ & -0.12 & -0.11 & -0.15 & -0.12 & -0.12 & -0.11 & -0.15 & -0.12 \\
\hline$T_{2}$ & 0.16 & 0.13 & 0.07 & 0.15 & 0.15 & 0.14 & 0.07 & 0.15 \\
\hline$T_{3}$ & 0.01 & 0.01 & 0.01 & 0.30 & 0.36 & 0.29 & 0.01 & 0.01 \\
\hline$T_{4}$ & 0.32 & 0.22 & 0.04 & 0.04 & 0.02 & 0.02 & 0.02 & 0.32 \\
\hline
\end{tabular}

Table 1: Anisotropy characterization of the textures $T_{1}, T_{2}, T_{3}$ and $T_{4}$ of figure 6

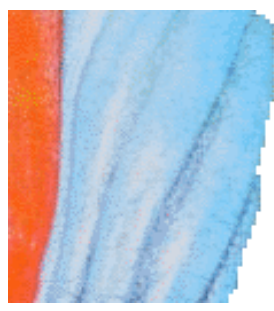

(a) $W_{1}$ : cheek

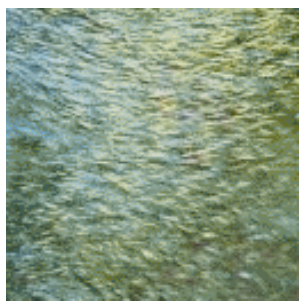

(b) $W_{2}$ : fur 1

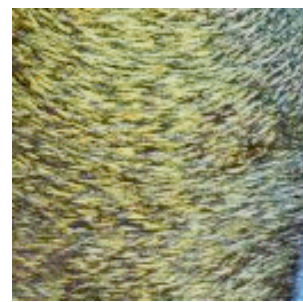

(c) $W_{3}$ : fur 2

Figure 7: Windows of the image Mandrill

\begin{tabular}{|l|c|l|l|l|l|l|l|l|}
\hline$d=4$ & $0^{\circ}$ & $22.5^{\circ}$ & $45^{\circ}$ & $67.5^{\circ}$ & $90^{\circ}$ & $112.5^{\circ}$ & $135^{\circ}$ & $157.5^{\circ}$ \\
\hline$W_{1}$ & 0.01 & 0.02 & 0.25 & 0.29 & 0.39 & 0.02 & 0.01 & 0.01 \\
\hline$W_{2}$ & 0.42 & 0.25 & 0.02 & 0.01 & 0.01 & 0.01 & 0.02 & 0.26 \\
\hline$W_{3}$ & 0.13 & 0.72 & 0.07 & 0.03 & 0.02 & 0.01 & 0.01 & 0.01 \\
\hline
\end{tabular}

Table 2: Anisotropy characterization of the windows $W_{1}, W_{2}$ and $W_{3}$ of figure 7

For the mandrill, we extracted three windows with a size $128 \times 128$ pixels. The figure 7.a corresponds to the mandrill cheeks which have been detected as homogeneous area, while figures 7.b and 7.c correspond to the fur of the mandrill which has been detected as heterogeneous area (see figure 2). The results of characterization by a measure of anisotropy are presented in table 2 .

We note that, for window $W_{1}$, for the direction $67.5^{\circ}$ and the close directions $45^{\circ}$ and $90^{\circ}$, we have very large coefficients. For the other directions, the coefficients are almost zero. The results for window $W_{2}$, with a horizontal dominant direction, were similar with only $0^{\circ}, 22.5^{\circ}$ and $157.5^{\circ}$ having large coefficients. For window $W_{3}$, for the direction $22.5^{\circ}$ and the close direction $0^{\circ}$, we have very large coefficients. For the other directions, the coefficients are almost zero. 


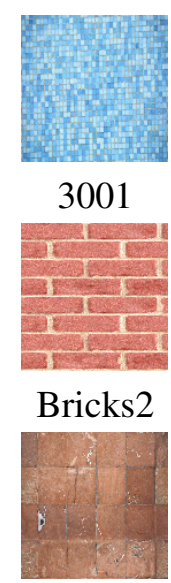

OldFloor

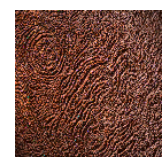

Arialphoto1

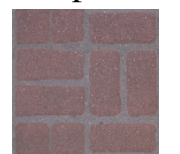

Brickwk

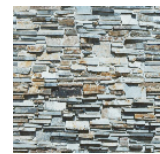

Stone041

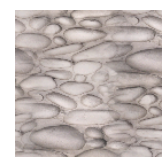

BigStones

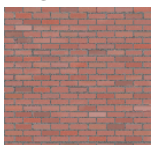

Brkwea_t

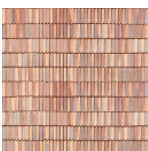

Stone291

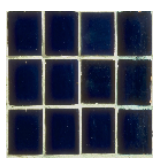

Blutile

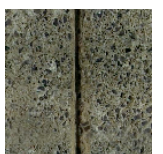

Cncrt3_t

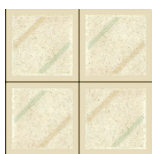

Stone421

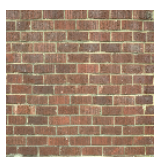

Brickm

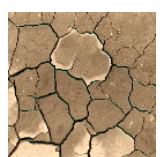

Cracks

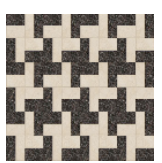

Stone451

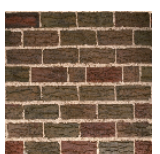

Brickm3

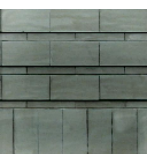

Gbrik3_t

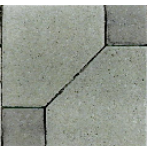

Tile1_t

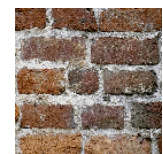

Bricks

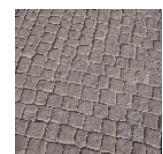

IMG0038

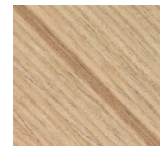

WOOD-bois4

Figure 8: Some textures available at http://textures. forrest.cz/

\begin{tabular}{|l|c|l|l|l|l|l|l|l|}
\hline$d=4$ & $0^{\circ}$ & $22.5^{\circ}$ & $45^{\circ}$ & $67.5^{\circ}$ & $90^{\circ}$ & $112.5^{\circ}$ & $135^{\circ}$ & $157.5^{\circ}$ \\
\hline 3001 & 0.01 & 0.41 & 0.03 & 0.07 & 0.38 & 0.03 & 0.00 & 0.02 \\
\hline Arialphoto1 & 0.05 & 0.04 & 0.78 & 0.03 & 0.00 & 0.02 & 0.02 & 0.06 \\
\hline BigStones & 0.35 & 0.29 & 0.12 & 0.02 & 0.02 & 0.00 & 0.15 & 0.03 \\
\hline Blutile & 0.43 & 0.03 & 0.04 & 0.01 & 0.44 & 0.01 & 0.00 & 0.01 \\
\hline Brickm & 0.46 & 0.02 & 0.05 & 0.00 & 0.42 & 0.00 & 0.00 & 0.01 \\
\hline Brickm3 & 0.49 & 0.01 & 0.00 & 0.01 & 0.45 & 0.00 & 0.01 & 0.01 \\
\hline Bricks & 0.50 & 0.03 & 0.04 & 0.00 & 0.40 & 0.02 & 0.00 & 0.00 \\
\hline Bricks2 & 0.49 & 0.03 & 0.02 & 0.00 & 0.41 & 0.02 & 0.01 & 0.01 \\
\hline Brickwk & 0.48 & 0.02 & 0.03 & 0.00 & 0.43 & 0.00 & 0.02 & 0.01 \\
\hline Brkwea_t & 0.46 & 0.00 & 0.01 & 0.01 & 0.46 & 0.02 & 0.01 & 0.00 \\
\hline Cncrt3_t & 0.03 & 0.00 & 0.02 & 0.01 & 0.88 & 0.01 & 0.04 & 0.00 \\
\hline Cracks & 0.03 & 0.06 & 0.24 & 0.05 & 0.27 & 0.23 & 0.04 & 0.05 \\
\hline Gbrik3_t & 0.55 & 0.02 & 0.02 & 0.01 & 0.38 & 0.01 & 0.00 & 0.00 \\
\hline IMG0038 & 0.03 & 0.01 & 0.02 & 0.45 & 0.02 & 0.01 & 0.02 & 0.43 \\
\hline OldFloor & 0.21 & 0.09 & 0.08 & 0.06 & 0.19 & 0.12 & 0.11 & 0.10 \\
\hline Stone041 & 0.63 & 0.02 & 0.02 & 0.01 & 0.24 & 0.02 & 0.03 & 0.02 \\
\hline Stone291 & 0.19 & 0.02 & 0.02 & 0.02 & 0.68 & 0.01 & 0.02 & 0.03 \\
\hline Stone421 & 0.34 & 0.03 & 0.14 & 0.03 & 0.36 & 0.02 & 0.03 & 0.03 \\
\hline Stone451 & 0.25 & 0.07 & 0.07 & 0.07 & 0.26 & 0.08 & 0.09 & 0.09 \\
\hline Tile1_t & 0.24 & 0.02 & 0.45 & 0.02 & 0.23 & 0.02 & 0.01 & 0.01 \\
\hline WOOD-bois4 & 0.01 & 0.01 & 0.01 & 0.01 & 0.01 & 0.05 & 0.85 & 0.04 \\
\hline
\end{tabular}

Table 3: Anisotropy characterization of the textures of figure 8

\section{Conclusion}

We proposed a new method that classifies the areas of a color image from the most homogeneous areas to the most heterogeneous areas. Thus, it is possible to create two sub-images, one of which contains the homogeneous areas, while the other color labeled layers contain the heterogeneous areas. We tested our method on tens of images and the classification according to the degree of homogeneity is always relevant. 
To our knowledge, there are no works related to the characterization of the anisotropy in color textures and there are no works related to the detection of homogeneous and heterogeneous areas in color images because "it is a difficult task to analyze the similarity of the colors and their distributions at the same time" (Deng [19]). Therefore, we cannot compare our approach with other works. As regards the textures, the majority of the existing methods are only one extension to color textures of the methods of gray level texture characterization. Very often, these methods work with each band of color, and are followed of a fusion step of the results. On the contrary, NCD matrices exploit actually the properties of the color spaces thanks to the perceptual difference $P D$ which depends to the color space.

The exploitation of the NCD matrices can provide information on a color image, such as the quantity of homogeneous and heterogeneous areas with their localization, the dominant colors, the dominant directions and the number of different textures. Therefore, our goal will be to seek groups of color labeled layers inside each class : in other words, the identification of different homogeneous areas in the homogeneous class and the number of different heterogeneous areas in the heterogeneous class (figure 4). This can be done by computing, for example, co-occurrence matrices between several color labeled layers in order to merge those which are similar. Thus, we have to study the connectivity of the pixels between the various color labeled layers. Integrated into a content-based image retrieval (CBIR), this information appears to be very useful.

Our future work will consist in exploiting NCD matrices in order to characterize, in a quantitative way, the color textures to extract some features and to define a texture representation space for the color images. In particular, how the detected main directions in a window can be taken into account to make a decision about the hypothesis that this window is a texture ? We also hope to develop a multi-scale analysis (by varying the size of the neighbourhood $d$ ) that could enable the detection of the edges between different textures [14].

\section{References}

[1] Sun C., Wee W. G., "Neighbouring gray level dependence matrix for texture classification", CVGIP, 23, 341-352, 1983.

[2] Berry J.R., Goutsias J., "A comparative study of matrix measures for maximum likelihood texture classification", IEEE Trans. SMC, 21(1), 252-261, 1991.

[3] Angulo J., Serra J., "Unified morphological color processing framework in a lum/sat/hue representation", Proceedings of the 7th International Symposium on Mathematical Morphology - ISMM'2005, 387-396, 2005.

[4] A. Hanbury, J. Serra, "Mathematical Morphology in the HLS Colour Space", Proceedings of the 12 British Machine Vision Conference, 451-460, 2001.

[5] A. Hanbury , J. Serra, "Morphological Operators on the Unit Circle", IEEE Transactions on Image Processing, 10(12): pp 1842-1850 December 2001.

[6] Smolarz A, "Evaluation de la dépendance spatiale locale pour la caractérisation de textures" [in french], 19eme colloque GRETSI sur le traitement du signal et des images, Paris, 8-11 Septembre 2003.

[7] B. Jacquin, A. Smolarz, "Characterization of the anisotropy in color textures", IEEE International Conference SITIS 2005, Cameroun, December 2005.

[8] B. Jacquin, A. Smolarz, "Color Space Comparison for Color Texture Analysis", 2005 Beijing International Conference on Imaging: Technology and Applications for the 21st Century, CSIST, Beijing, 22-28 May 2005 .

[9] A. R. Rao, G. L. Lohse, "Towards a texture naming system: identifying relevant dimensions of texture", VIS '93: Proceedings of the 4th conference on Visualization, pp 220-227, San Jose, California, 1993. 
[10] A. R. Rao, B. G. Schunck, "Computing oriented texture fields", CVGIP: Graphical Models and Image Processing, v. 53, num. 2, pp 157-185, March 1991.

[11] A. R. Rao, R. C. Jain, "Computerized Flow Field Analysis: Oriented Texture Fields", IEEE Transactions on Pattern Analysis and Machine Intelligence, v.14, num. 7, pp 693-709, July 1992.

[12] P. Brodatz, ’Textures: a photographic album for artists and designers", Dover publications, New York, 1966.

[13] A. Mojsilovic, J. Kovacevic, J. Hu, R. J. Safranek, K. Ganapathy, "Retrieval of Color Patterns Based on Perceptual Dimensions of Texture and Human Similarity Rules", Proc. 1999 SPIE Human Vision and Electronic Imaging, vol. 3644, San Jose, January 1999.

[14] C. Germain, J.P. DaCosta, P. Baylou, "Multiscale Estimation of Textural Features, Application to the Characterization of Texture Anisotropy", ICPR 00, 2000.

[15] C. F. Shu, R. C. Jain, "Vector Field Analysis for Oriented Patterns”, IEEE Transactions on Pattern Analysis and Machine Intelligence, vol. 16, num. 9, pp 946-950, 1994.

[16] M. P. Almeida, "Anisotropic Textures with Arbitrary Orientation", Journal of Mathematical Imaging and Vision, Vol. 7, Issue 3, pp 241-251, June 1997.

[17] M. Fontaine, L. Macaire, J.-G. Postaire, "Image segmentation based on an original multiscale analysis of the pixel connectivity properties", IEEE International Conference on Image Processing, Vancouver, Canada, vol.1, pp 804-807, 2000.

[18] J. Huang, S. R. Kumar, M. Mitra, W. J. Zhu, R. Zabih, "Image Indexing Using Color Correlograms", Proc. IEEE Int. Conf. on Computer Vision and Pattern Recognition, pp 762-768, 1997.

[19] Y. Deng, B.S. Manjunath, "Unsupervised Segmentation of Color-Texture Regions in Images and Video", IEEE Trans. On Pattern Analysis and Machine Intelligence, vol. 23, num. 8, August 2001.

[20] C. Montagne, S. Lelandais, A. Smolarz, Ph. Cornu, M. C. Larabi, C. Fernandez-Maloigne, ”Adaptative Color Quantization Using the Baker's Transformation”, Journal of Electronic Imaging, Vol. 15(2) 2006. 